\title{
Article \\ On $g$-Noncommuting Graph of a Finite Group Relative to Its Subgroups
}

\author{
Monalisha Sharma ${ }^{1}$, Rajat Kanti Nath ${ }^{1, *}$ and Yilun Shang ${ }^{2, *(D)}$ \\ 1 Department of Mathematical Sciences, Tezpur University, Tezpur 784028, India; \\ monalishasharma2013@gmail.com \\ 2 Department of Computer and Information Sciences, Northumbria University, Newcastle NE1 8ST, UK \\ * Correspondence: rajatkantinath@yahoo.com (R.K.N.); yilun.shang@northumbria.ac.uk (Y.S.)
}

check for updates

Citation: Sharma, M.; Nath, R.K.; Shang, Y. On $g$-Noncommuting Graph of a Finite Group Relative to Its Subgroups. Mathematics 2021, 9 , 3147. https://doi.org/10.3390/ math9233147

Academic Editor: Adolfo Ballester-Bolinches

Received: 9 November 2021 Accepted: 3 December 2021 Published: 6 December 2021

Publisher's Note: MDPI stays neutral with regard to jurisdictional claims in published maps and institutional affiliations.

Copyright: (c) 2021 by the authors. Licensee MDPI, Basel, Switzerland. This article is an open access article distributed under the terms and conditions of the Creative Commons Attribution (CC BY) license (https:// creativecommons.org/licenses/by/ $4.0 /)$.

\begin{abstract}
Let $H$ be a subgroup of a finite non-abelian group $G$ and $g \in G$. Let $Z(H, G)=\{x \in H$ : $x y=y x, \forall y \in G\}$. We introduce the graph $\Delta_{H, G}^{g}$ whose vertex set is $G \backslash Z(H, G)$ and two distinct vertices $x$ and $y$ are adjacent if $x \in H$ or $y \in H$ and $[x, y] \neq g, g^{-1}$, where $[x, y]=x^{-1} y^{-1} x y$. In this paper, we determine whether $\Delta_{H, G}^{g}$ is a tree among other results. We also discuss about its diameter and connectivity with special attention to the dihedral groups.
\end{abstract}

Keywords: finite group; g-noncommuting graph; connected graph

\section{Introduction}

Several properties of groups can be described through properties of graphs and vice versa. Characterizations of finite groups through various graphs defined on them have been an interesting topic of research over the last five decades. The non-commuting graph is one of such interesting graphs widely studied in the literature [1-16] since its inception [17]. In this paper, we introduce a generalization of non-commuting graph of a finite group. Let $H$ be a subgroup of a finite non-abelian group $G$ and $g \in G$. Let $Z(H, G)=\{x \in H: x y=y x, \forall y \in G\}$. We introduce the graph $\Delta_{H, G}^{g}$ whose vertex set is $G \backslash Z(H, G)$ and two distinct vertices $x$ and $y$ are adjacent if $x \in H$ or $y \in H$ and $[x, y] \neq g, g^{-1}$, where $[x, y]=x^{-1} y^{-1} x y$. Clearly, $\Delta_{H, G}^{g}=\Delta_{H, G}^{g^{-1}}$. In addition, $\Delta_{H, G}^{g}$ is an induced subgraph of $\Gamma_{H, G}^{g}$, studied by the authors in [18], induced by $G \backslash Z(H, G)$. If $H=G$ and $g=1$, then $\Delta_{H, G}^{g}:=\Gamma_{G}$, the non-commuting graph of $G$. If $H=G$, then $\Delta_{H, G}^{g}:=\Delta_{G}^{g}$, a generalization of $\Gamma_{G}$ called an induced g-noncommuting graph of $G$ on $G \backslash Z(G)$ studied extensively in [19-21] by Erfanian and his collaborators.

If $g \notin K(H, G):=\{[x, y]: x \in H$ and $y \in G\}$, then any pair of vertices $(x, y)$ are adjacent in $\Delta_{H, G}^{g}$ trivially if $x, y \in H$ or one of $x$ and $y$ belongs to $H$. Therefore, we consider $g \in K(H, G)$. In addition, if $H=Z(H, G)$, then $K(H, G)=\{1\}$ and so $g=1$. Thus, throughout this paper, we shall consider $H \neq Z(H, G)$ and $g \in K(H, G)$. In this paper, we determine whether $\Delta_{H, G}^{g}$ is a tree among other results. We also discuss its diameter and connectivity with special attention to the dihedral groups. We conclude this section by the following examples of $\Delta_{H, G}^{g}$, where $G=A_{4}=\left\langle a, b: a^{2}=b^{3}=(a b)^{3}=1\right\rangle$ and the subgroup $H$ is given by $H_{1}=\{1, a\}, H_{2}=\left\{1, b a b^{2}\right\}$ or $H_{3}=\left\{1, b^{2} a b\right\}$ (see Figures 1-6). 


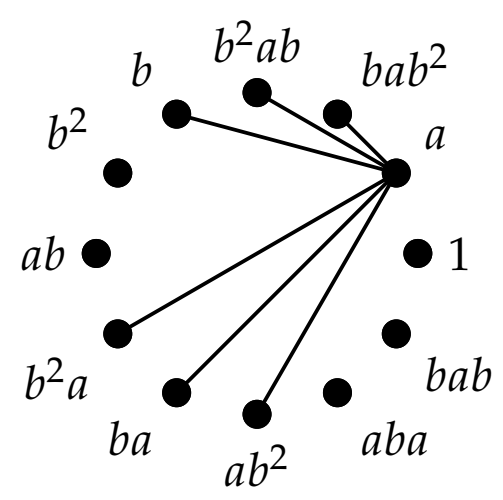

Figure 1. $\Delta_{H_{1}, A_{4}}^{b a b^{2}}$.

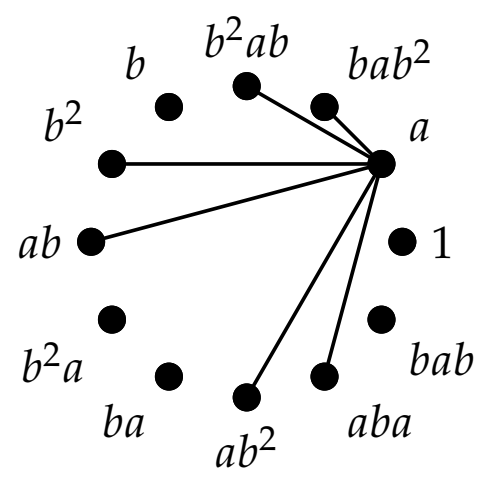

Figure 2. $\Delta_{H_{1}, A_{4}}^{b^{2} a b}$.

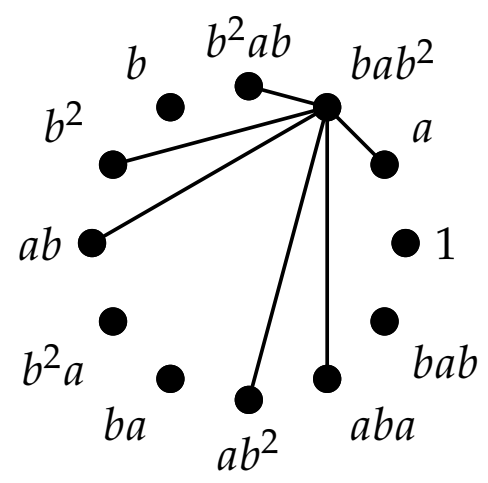

Figure 3. $\Delta_{H_{2}, A_{4}}^{a}$.

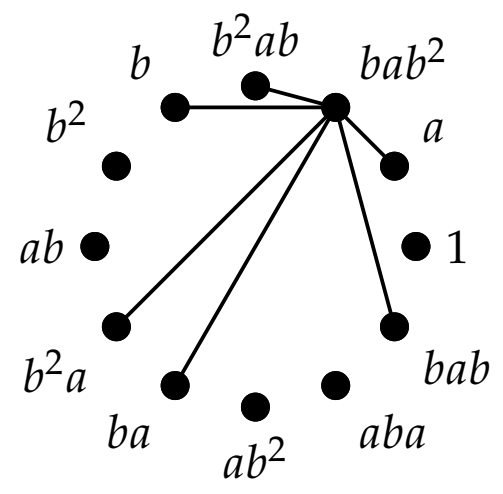

Figure 4. $\Delta_{H_{2}, A_{4}}^{b^{2} a b}$. 


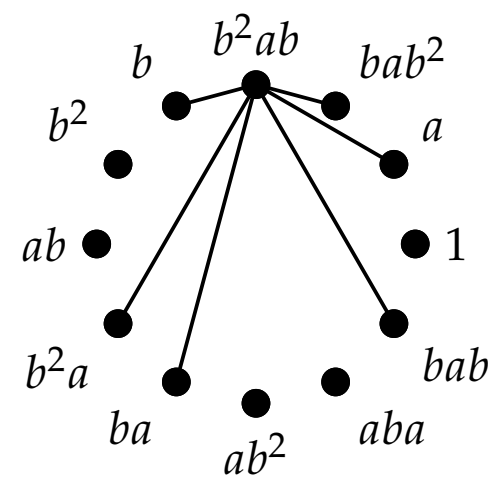

Figure 5. $\Delta_{H_{3}, A_{4}}^{a}$.

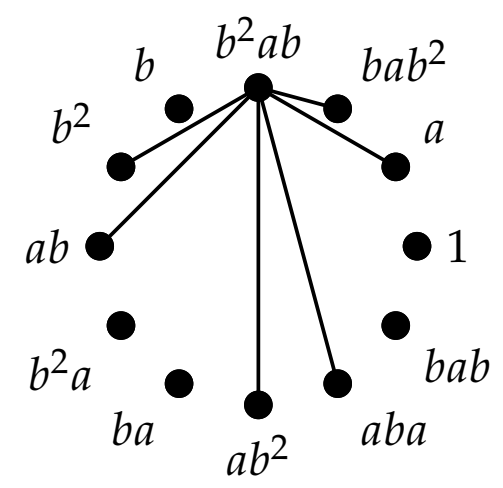

Figure 6. $\Delta_{H_{3}, A_{4}}^{b a b^{2}}$.

\section{Vertex Degree and a Consequence}

In this section, we first determine $\operatorname{deg}(x)$, the degree of a vertex $x$ of the graph $\Delta_{H, G}^{g}$. After that, we determine whether $\Delta_{H, G}^{g}$ is a tree. Corresponding to Theorems 2.1 and 2.2 of [18], we have the following two results for $\Delta_{H, G}^{g}$.

Theorem 1. Let $x \in H \backslash Z(H, G)$ be any vertex in $\Delta_{H, G}^{g}$.

(a) If $g=1$, then $\operatorname{deg}(x)=|G|-\left|C_{G}(x)\right|$.

(b) If $g \neq 1$ and $g^{2} \neq 1$, then

$$
\operatorname{deg}(x)= \begin{cases}|G|-|Z(H, G)|-\left|C_{G}(x)\right|-1, & \text { if } x \text { is conjugate to } \\ |G|-|Z(H, G)|-2\left|C_{G}(x)\right|-1, & \text { if } x \text { is conjugate to } x g^{-1} \\ & x g \text { and } x g^{-1} .\end{cases}
$$

(c) If $g \neq 1$ and $g^{2}=1$, then $\operatorname{deg}(x)=|G|-|Z(H, G)|-\left|C_{G}(x)\right|-1$, whenever $x$ is conjugate to $x g$.

Proof. (a) Let $g=1$. Then, $\operatorname{deg}(x)$ is the number of $y \in G \backslash Z(H, G)$ such that $x y \neq y x$. Hence,

$$
\operatorname{deg}(x)=|G|-|Z(H, G)|-\left(\left|C_{G}(x)\right|-|Z(H, G)|\right)=|G|-\left|C_{G}(x)\right|
$$

Proceeding as the proof of (Theorem 2.1 (b), (c), [18]), parts (b) and (c) follow noting that the vertex set of $\Delta_{H, G}^{g}$ is $G \backslash Z(H, G)$.

Theorem 2. Let $x \in G \backslash H$ be any vertex in $\Delta_{H, G}^{g}$.

(a) If $g=1$, then $\operatorname{deg}(x)=|H|-\left|C_{H}(x)\right|$. 
(b)

$$
\begin{aligned}
& \text { If } g \neq 1 \text { and } g^{2} \neq 1 \text {, then } \\
& \operatorname{deg}(x)=\left\{\begin{aligned}
&|H|-|Z(H, G)|-\left|C_{H}(x)\right|, \text { if } x \text { is conjugate to } x g \text { or } \\
& x g^{-1} \text { for some element in } H \\
&|H|-|Z(H, G)|-2\left|C_{H}(x)\right|, \text { if } x \text { is conjugate to } x g \text { and } \\
& x g^{-1} \text { for some element in } H .
\end{aligned}\right.
\end{aligned}
$$

(c) If $g \neq 1$ and $g^{2}=1$, then $\operatorname{deg}(x)=|H|-|Z(H, G)|-\left|C_{H}(x)\right|$, whenever $x$ is conjugate to $x g$, for some element in $\mathrm{H}$.

Proof. (a) Let $g=1$. Then, $\operatorname{deg}(x)$ is the number of $y \in H \backslash Z(H, G)$ such that $x y \neq y x$. Hence,

$$
\operatorname{deg}(x)=|H|-|Z(H, G)|-\left(\left|C_{H}(x)\right|-|Z(H, G)|\right)=|H|-\left|C_{H}(x)\right| .
$$

Proceeding as the proof of (Theorem 2.2 (b), (c), [18]), parts (b) and (c) follow noting that the vertex set of $\Delta_{H, G}^{g}$ is $G \backslash Z(H, G)$.

As a consequence of the above results, we have the following:

Theorem 3. If $|H| \neq 2,3,4,6$, then $\Delta_{H, G}^{g}$ is not a tree.

Proof. Suppose that $\Delta_{H, G}^{g}$ is a tree. Then, there exists a vertex $x \in G \backslash Z(H, G)$ such that $\operatorname{deg}(x)=1$. If $x \in H \backslash Z(H, G)$, then we have the following cases.

Case 1: If $g=1$, then by Theorem $1(\mathrm{a})$, we have $\operatorname{deg}(x)=|G|-\left|C_{G}(x)\right|=1$. Therefore, $\left|C_{G}(x)\right|=1$, contradiction.

Case 2: If $g \neq 1$ and $g^{2}=1$, then by Theorem 1 (c), we have $\operatorname{deg}(x)=|G|-|Z(H, G)|-$ $\left|C_{G}(x)\right|-1=1$. That is,

$$
|G|-|Z(H, G)|-\left|C_{G}(x)\right|=2 .
$$

Therefore, $|Z(H, G)|=1$ or 2 . Thus, (1) gives $|G|-\left|C_{G}(x)\right|=3$ or 4 . Therefore, $|G|=6$ or 8 . Since $|H| \neq 2,3,4,6$, we must have $G \cong D_{8}$ or $Q_{8}$ and $H=G$ and hence, by (Theorem 2.5, [22]), we get a contradiction.

Case 3: If $g \neq 1$ and $g^{2} \neq 1$, then by Theorem $1($ b), we have $\operatorname{deg}(x)=|G|-|Z(H, G)|-$ $\left|C_{G}(x)\right|-1=1$, which will lead to (1) (and eventually to a contradiction) or $\operatorname{deg}(x)=$ $|G|-|Z(H, G)|-2\left|C_{G}(x)\right|-1=1$. That is,

$$
\text { or }|G|-|Z(H, G)|-2\left|C_{G}(x)\right|=2 \text {. }
$$

Therefore, $|Z(H, G)|=1$ or 2 . Thus, if $|Z(H, G)|=1$, then (2) gives $|G|=9$, which is a contradiction since $G$ is non-abelian. Again, if $|Z(H, G)|=2$, then (2) gives $\left|C_{G}(x)\right|=2$ or 4. Therefore, $|G|=8$ or $|G|=12$. If $|G|=8$, then we get a contradiction as shown in Case 2 above. If $|G|=12$, then $G \cong D_{12}$ or $Q_{12}$, since $|Z(H, G)|=2$. In both of the cases, we must have $H=G$ and hence, by (Theorem 2.5, [22]), we get a contradiction.

Now, we assume that $x \in G \backslash H$ and consider the following cases.

Case 1: If $g=1$, then by Theorem 2(a), we have $\operatorname{deg}(x)=|H|-\left|C_{H}(x)\right|=1$. Therefore, $|H|=2$, a contradiction.

Case 2: If $g \neq 1$ and $g^{2}=1$, then by Theorem 2(c), we have $\operatorname{deg}(x)=|H|-|Z(H, G)|-$ $\left|C_{H}(x)\right|=1$. That is,

$$
|H|-\left|C_{H}(x)\right|=2 \text {. }
$$

Therefore, $|H|=3$ or 4 , a contradiction.

Case 3: If $g \neq 1$ and $g^{2} \neq 1$, then by Theorem 2(b), we have $\operatorname{deg}(x)=|H|-|Z(H, G)|-$ $\left|C_{H}(x)\right|=1$, which leads to (3) or $\operatorname{deg}(x)=|H|-|Z(H, G)|-2\left|C_{H}(x)\right|=1$. That is,

$$
|H|-2\left|C_{H}(x)\right|=2 \text {. }
$$


Therefore, $\left|C_{H}(x)\right|=1$ or 2 . Thus, if $\left|C_{H}(x)\right|=1$, then (4) gives $|H|=4$, a contradiction. If $\left|C_{H}(x)\right|=2$, then (4) gives $|H|=6$, a contradiction.

The following theorems also show that the conditions on $|H|$ as mentioned in Theorem 3 can not be removed completely.

Theorem 4. If $G$ is a non-abelian group of order $\leq 12$ and $g=1$, then $\Delta_{H, G}^{g}$ is a tree if and only if $G \cong D_{6}$ or $D_{10}$ and $|H|=2$.

Proof. If $H$ is the trivial subgroup of $G$, then $\Delta_{H, G}^{g}$ is an empty graph. If $H=G$, then, by (Theorem 2.5, [22]), we have $\Delta_{H, G}^{g}$ is not a tree. Thus, we examine only the proper subgroups of $G$, where $G \cong D_{6}, D_{8}, Q_{8}, D_{10}, D_{12}, Q_{12}$ or $A_{4}$. We consider the following cases:

Case 1: $G \cong D_{6}=\left\langle a, b: a^{3}=b^{2}=1\right.$ and $\left.b a b^{-1}=a^{-1}\right\rangle$. If $|H|=2$, then $H=\langle x\rangle$, where $x=b, a b$ and $a^{2} b$. We have $[x, y] \neq 1$ for all $y \in G \backslash Z(H, G)$. Therefore, $\Delta_{H, D_{6}}^{g}$ is a star graph and hence a tree. If $|H|=3$, then $H=\left\{1, a, a^{2}\right\}$. In this case, the vertices $a, a b, a^{2}$ and $b$ make a cycle since $[a b, a]=a^{2}=\left[a^{2}, a b\right]$ and $[a, b]=a=\left[b, a^{2}\right]$.

Case 2: $G \cong D_{8}=\left\langle a, b: a^{4}=b^{2}=1\right.$ and $\left.b a b^{-1}=a^{-1}\right\rangle$. If $|H|=2$, then $H=Z\left(D_{8}\right)$ or $\left\langle a^{r} b\right\rangle$, where $r=1,2,3,4$. Clearly $\Delta_{H, D_{8}}^{g}$ is an empty graph if $H=Z\left(D_{8}\right)$. If $H=\left\langle a^{r} b\right\rangle$, then, in each case, $a^{2}$ is an isolated vertex in $G \backslash H$ (since $\left[a^{2}, a^{r} b\right]=1$ ). Hence, $\Delta_{H, D_{8}}^{g}$ is disconnected. If $|H|=4$, then $H=\left\{1, a, a^{2}, a^{3}\right\},\left\{1, a^{2}, b, a^{2} b\right\}$ or $\left\{1, a^{2}, a b, a^{3} b\right\}$. If $H=\left\{1, a, a^{2}, a^{3}\right\}$, then, the vertices $a b, a, b$, and $a^{3}$ make a cycle; if $H=\left\{1, a^{2}, b, a^{2} b\right\}$, then the vertices $a b, b, a^{3}$ and $a^{2} b$ make a cycle, and, if $H=\left\{1, a^{2}, a b, a^{3} b\right\}$, then the vertices $a b$, $a, a^{3} b$ and $b$ make a cycle (since $[a, b]=\left[a^{3}, b\right]=\left[a^{3}, a b\right]=\left[a^{3}, a^{2} b\right]=[a b, a]=\left[a^{2} b, a b\right]=$ $\left.\left[a^{3} b, a\right]=[b, a b]=\left[b, a^{3} b\right]=a^{2} \neq 1\right)$.

Case 3: $G \cong Q_{8}=\left\langle a, b: a^{4}=1, b^{2}=a^{2}\right.$ and $\left.b a b^{-1}=a^{-1}\right\rangle$. If $|H|=2$, then $H=Z\left(Q_{8}\right)$ and so $\Delta_{H, Q_{8}}^{g}$ is an empty graph. If $|H|=4$, then $H=\left\{1, a, a^{2}, a^{3}\right\}$, $\left\{1, a^{2}, b, a^{2} b\right\}$ and $\left\{1, a^{2}, a b, a^{3} b\right\}$. Again, if $H=\left\{1, a, a^{2}, a^{3}\right\}$, then the vertices $a, b, a^{3}$ and $a b$ make a cycle; if $H=\left\{1, a^{2}, b, a^{2} b\right\}$, then the vertices $b, a^{3} b, a^{2} b$ and $a^{3}$ make a cycle; and if $H=\left\{1, a^{2}, a b, a^{3} b\right\}$, then the vertices $a b, a, a^{3} b$ and $a^{2} b$ make a cycle (since $[a, b]=$ $\left.\left[b, a^{3}\right]=\left[a^{3}, a b\right]=[a b, a]=\left[b, a^{3} b\right]=\left[a^{3} b, a^{2} b\right]=\left[a^{2} b, a^{3}\right]=\left[a, a^{3} b\right]=\left[a^{2} b, a b\right]=a^{2} \neq 1\right)$.

Case 4: $G \cong D_{10}=\left\langle a, b: a^{5}=b^{2}=1\right.$ and $\left.b a b^{-1}=a^{-1}\right\rangle$. If $|H|=2$, then $H=\left\langle a^{r} b\right\rangle$, for every integer $r$ such that $1 \leq r \leq 5$. For each case of $H, \Delta_{H, D_{10}}^{g}$ is a star graph since $\left[a^{r} b, x\right] \neq g$ for all $x \in G \backslash H$. If $|H|=5$, then $H=\left\{1, a, a^{2}, a^{3}, a^{4}\right\}$. In this case, the vertices $a, a b, a^{3}$ and $a^{3} b$ make a cycle in $\Delta_{H, D_{10}}^{g}$ since $[a, a b]=a^{3} \neq 1,\left[a b, a^{3}\right]=a \neq 1$, $\left[a^{3}, a^{3} b\right]=a^{4} \neq 1$ and $\left[a^{3} b, a\right]=a^{2} \neq 1$.

Case 5: $G \cong D_{12}=\left\langle a, b: a^{6}=b^{2}=1\right.$ and $\left.b a b^{-1}=a^{-1}\right\rangle$. If $|H|=2$, then $H=Z\left(D_{12}\right)$ or $\left\langle a^{r} b\right\rangle$, for every integer $r$ such that $1 \leq r \leq 6$. If $|H|=3$, then $H=\left\{1, a^{2}, a^{4}\right\}$. If $|H|=4$, then $H=\left\{1, a^{3}, b, a^{3} b\right\},\left\{1, a^{3}, a b, a^{4} b\right\}$ or $\left\{1, a^{3}, a^{2} b, a^{5} b\right\}$. If $|H|=6$, then $H=$ $\left\{1, a, a^{2}, a^{3}, a^{4}, a^{5}\right\},\left\{1, a^{2}, a^{4}, b, a^{2} b, a^{4} b\right\}$ or $\left\{1, a^{2}, a^{4}, a b, a^{3} b, a^{5} b\right\}$. Note that $\Delta_{H, D_{12}}^{g}$ is an empty graph if $H=Z\left(D_{12}\right)$. If $H=\left\langle a^{r} b\right\rangle$ (for $1 \leq r \leq 6$ ), $\left\{1, a^{2}, a^{4}\right\},\left\{1, a^{2}, a^{4}, b, a^{2} b, a^{4} b\right\}$ or $\left\{1, a^{2}, a^{4}, a b, a^{3} b, a^{5} b\right\}$, then, in each case, the vertex $a^{3}$ is an isolated vertex in $G \backslash H$ (since $a^{3} \in Z\left(D_{12}\right)$ ) and hence $\Delta_{H, D_{12}}^{g}$ is disconnected. We have $[a, b]=\left[b, a^{5}\right]=[a, a b]=$ $\left[a^{4}, a^{4} b\right]=\left[a^{5} b, a^{2}\right]=\left[b, a^{2}\right]=\left[a^{2} b, a^{5}\right]=\left[a^{3} b, a^{2}\right]=\left[a^{3} b, a^{5}\right]=a^{4} \neq 1$ and $\left[a^{5}, a^{5} b\right]=$ $\left[a^{5} b, a\right]=\left[a b, a^{4}\right]=\left[a^{4} b, a\right]=\left[a^{2}, a^{2} b\right]=\left[a^{2} b, a\right]=a^{2} \neq 1$. Therefore, if $H=\left\{1, a^{3}, b, a^{3} b\right\}$, then the vertices $b, a^{2}, a^{3} b$ and $a^{5}$ make a cycle; if $H=\left\{1, a^{3}, a b, a^{4} b\right\}$, then the vertices $a, a b$, $a^{4}$ and $a^{4} b$ make a cycle; if $H=\left\{1, a^{3}, a^{2} b, a^{5} b\right\}$, then the vertices $a^{2}, a^{2} b, a^{5}$ and $a^{5} b$ make a cycle; and if $H=\left\{1, a, a^{2}, a^{3}, a^{4}, a^{5}\right\}$, then the vertices $a, b, a^{2}$ and $a^{2} b$ make a cycle.

Case 6: $G \cong A_{4}=\left\langle a, b: a^{2}=b^{3}=(a b)^{3}=1\right\rangle$. If $|H|=2$, then $H=\langle a\rangle,\left\langle b a b^{2}\right\rangle$ or $\left\langle b^{2} a b\right\rangle$. Since the elements $a, b a b^{2}$ and $b^{2} a b$ commute among themselves, in each case the remaining two elements in $G \backslash H$ remain isolated and hence $\Delta_{H, A_{4}}^{g}$ is disconnected. If $|H|=3$, then $H=\langle x\rangle$, where $x=b, a b, b a, a b a$. In each case, the vertices $x, a, x^{-1}$ and $b a b^{2}$ make a cycle. 
If $|H|=4$, then $H=\left\{1, a, b a b^{2}, b^{2} a b\right\}$. In this case, the vertices $a, b, b a b^{2}$ and $a b$ make a cycle.

Case 7: $G \cong Q_{12}=\left\langle a, b: a^{6}=1, b^{2}=a^{3}\right.$ and $\left.b a b^{-1}=a^{-1}\right\rangle$. If $|H|=2$, then $H=Z\left(Q_{12}\right)$ and so $\Delta_{H, Q_{12}}^{g}$ is an empty graph. If $|H|=3$, then $H=\left\{1, a^{2}, a^{4}\right\}$. In this case, $a^{3}$ is an isolated vertex in $G \backslash H$ (since $a^{3} \in Z\left(Q_{12}\right)$ ) and so $\Delta_{H, Q_{12}}^{g}$ is disconnected. If $|H|=4$, then $H=\left\{1, a^{3}, b, a^{3} b\right\},\left\{1, a^{3}, a b, a^{4} b\right\}$ or $\left\{1, a^{3}, a^{2} b, a^{5} b\right\}$. If $|H|=6$, then $H=\left\{1, a, a^{2}, a^{3}, a^{4}, a^{5}\right\}$. We have $[a, b]=[a, a b]=\left[a^{4}, a^{4} b\right]=\left[a^{5} b, a^{2}\right]=\left[b, a^{2}\right]=\left[b, a^{5}\right]=$ $\left[a^{2} b, a^{5}\right]=\left[a^{3} b, a^{2}\right]=\left[a^{3} b, a^{5}\right]=a^{4} \neq 1$ and $\left[a^{5}, a^{5} b\right]=\left[a^{5} b, a\right]=\left[a b, a^{4}\right]=\left[a^{4} b, a\right]=$ $\left[a^{2}, a^{2} b\right]=\left[a^{2} b, a\right]=a^{2} \neq 1$. Therefore, if $H=\left\{1, a^{3}, b, a^{3} b\right\}$, then the vertices $a^{2}, b, a^{5}$ and $a^{3} b$ make a cycle; if $H=\left\{1, a^{3}, a b, a^{4} b\right\}$, then the vertices $a, a b, a^{4}$ and $a^{4} b$ make a cycle; if $H=\left\{1, a^{3}, a^{2} b, a^{5} b\right\}$, then the vertices $a^{2}, a^{2} b, a^{5}$ and $a^{5} b$ make a cycle; and if $H=\left\{1, a, a^{2}, a^{3}, a^{4}, a^{5}\right\}$, then the vertices $a, b, a^{2}$ and $a^{2} b$ make a cycle. This completes the proof.

Theorem 5. If $G$ is a non-abelian group of order $\leq 12$ and $g \neq 1$, then $\Delta_{H, G}^{g}$ is a tree if and only if $g^{2}=1, G \cong A_{4}$ and $|H|=2$ such that $H=\langle g\rangle$.

Proof. If $H$ is the trivial subgroup of $G$, then $\Delta_{H, G}^{g}$ is an empty graph. If $H=G$, then, by (Theorem 2.5, [22]), we have $\Delta_{H, G}^{g}$ is not a tree. Thus, we examine only the proper subgroups of $G$, where $G \cong D_{6}, D_{8}, Q_{8}, D_{10}, D_{12}, Q_{12}$, or $A_{4}$. We consider the following two cases.

Case 1: $g^{2}=1$

In this case, $G \cong D_{8}, Q_{8}$ or $A_{4}$. If $G \cong D_{8}=\left\langle a, b: a^{4}=b^{2}=1\right.$ and $\left.b a b^{-1}=a^{-1}\right\rangle$, then $g=a^{2}$ and $|H|=2$, 4. If $|H|=2$, then $H=Z\left(D_{8}\right)$ or $\left\langle a^{r} b\right\rangle$, for every integer $r$ such that $1 \leq r \leq 4$. For $H=Z\left(D_{8}\right), \Delta_{H, D_{8}}^{g}$ is an empty graph. For $H=\left\langle a^{r} b\right\rangle$, in each case, $a$ is an isolated vertex in $G \backslash H$ (since $\left[a, a^{r} b\right]=a^{2}$ ) and hence $\Delta_{H, D_{8}}^{g}$ is disconnected. If $|H|=4$, then $H=\left\{1, a, a^{2}, a^{3}\right\},\left\{1, a^{2}, b, a^{2} b\right\}$ or $\left\{1, a^{2}, a b, a^{3} b\right\}$. For $H=\left\{1, a, a^{2}, a^{3}\right\}, b$ is an isolated vertex in $G \backslash H$ (since $[a, b]=a^{2}=\left[a^{3}, b\right]$ ) and hence $\Delta_{H, D_{8}}^{g}$ is disconnected. If $H=\left\{1, a^{2}, b, a^{2} b\right\}$ or $\left\{1, a^{2}, a b, a^{3} b\right\}$, then $a$ is an isolated vertex in $G \backslash H$ (since $\left[a, a^{r} b\right]=a^{2}$ for every integer $r$ such that $1 \leq r \leq 4)$ and hence $\Delta_{H, D_{8}}^{g}$ is disconnected.

If $G \cong Q_{8}=\left\langle a, b: a^{4}=1, b^{2}=a^{2}\right.$ and $\left.b a b^{-1}=a^{-1}\right\rangle$, then $g=a^{2}$ and $|H|=2,4$. If $|H|=2$, then $H=Z\left(Q_{8}\right)$ and hence $\Delta_{H, Q_{8}}^{g}$ is an empty graph. If $|H|=4$, then $H=\left\{1, a, a^{2}, a^{3}\right\},\left\{1, a^{2}, b, a^{2} b\right\}$ or $\left\{1, a^{2}, a b, a^{3} b\right\}$. In each case, vertices of $H \backslash Z(H, G)$ commute with each other and commutator of these vertices and those of $G \backslash H$ equals $a^{2}$. Hence, the vertices in $G \backslash H$ remain isolated and so $\Delta_{H, Q_{8}}^{g}$ is disconnected.

If $G \cong A_{4}=\left\langle a, b: a^{2}=b^{3}=(a b)^{3}=1\right\rangle$, then $g \in\left\{a, b a b^{2}, b^{2} a b\right\}$ and $|H|=2,3,4$. If $|H|=2$, then $H=\langle a\rangle,\left\langle b a b^{2}\right\rangle$ or $\left\langle b^{2} a b\right\rangle$. If $H=\langle g\rangle$, then $\Delta_{H, A_{4}}^{g}$ is a star graph because $[g, x] \neq g$ for all $x \in G \backslash H$ and hence a tree; otherwise, $\Delta_{H, A_{4}}^{g}$ is not a tree as shown in Figures 1-6. If $|H|=3$, then $H=\langle x\rangle$, where $x=b, a b, b a, a b a$ or their inverses. We have $\left[x, x^{-1}\right]=1,[x, g] \neq g$ and $\left[x^{-1}, g\right] \neq g$. Therefore, $x, x^{-1}$ and $g$ make a triangle for each such subgroup in the graph $\Delta_{H, A_{4}}^{g}$. If $|H|=4$, then $H=\left\{1, a, b a b^{2}, b^{2} a b\right\}$. Since $H$ is abelian, the vertices $a, b a b^{2}$ and $b^{2} a b$ make a triangle in the graph $\Delta_{H, A_{4}}^{g}$.

Case 2: $g^{2} \neq 1$

In this case, $G \cong D_{6}, D_{10}, D_{12}$ or $Q_{12}$.

If $G \cong D_{6}=\left\langle a, b: a^{3}=b^{2}=1\right.$ and $\left.b a b^{-1}=a^{-1}\right\rangle$, then $g \in\left\{a, a^{2}\right\}$ and $|H|=2,3$. We have $\Delta_{H, D_{6}}^{a}=\Delta_{H, D_{6}}^{a^{2}}$ since $a^{-1}=a^{2}$. If $|H|=2$, then $H=\langle x\rangle$, where $x=b$, $a b$ and $a^{2} b$. We have $[x, y] \in\left\{g, g^{-1}\right\}$ for all $y \in G \backslash H$ and so $\Delta_{H, D_{6}}^{g}$ is an empty graph. If $|H|=3$, then $H=\left\{1, a, a^{2}\right\}$. In this case, the vertices of $G \backslash H$ remain isolated since, for $y \in G \backslash H$, we have $[a, y],\left[a^{2}, y\right] \in\left\{g, g^{-1}\right\}$.

If $G \cong D_{10}=\left\langle a, b: a^{5}=b^{2}=1\right.$ and $\left.b a b^{-1}=a^{-1}\right\rangle$, then $g \in\left\{a, a^{2}, a^{3}, a^{4}\right\}$ and $|H|=2,5$. We have $\Delta_{H, D_{10}}^{a}=\Delta_{H, D_{10}}^{a^{4}}$ and $\Delta_{H, D_{10}}^{a^{2}}=\Delta_{H, D_{10}}^{a^{3}}$ since $a^{-1}=a^{4}$ and $\left(a^{2}\right)^{-1}=a^{3}$. 
Suppose that $|H|=2$. Then, $H=\left\langle a^{r} b\right\rangle$, for every integer $r$ such that $1 \leq r \leq 5$. If $g=a$, then for each subgroup $H, a^{2}$ is an isolated vertex in $\Delta_{H, D_{10}}^{g}$ (since $\left[a^{2}, a^{r} b\right]=a^{4}$ for every integer $r$ such that $1 \leq r \leq 5$ ). If $g=a^{2}$, then for each subgroup $H, a$ is an isolated vertex in $\Delta_{H, D_{10}}^{g}$ (since $\left[a, a^{r} b\right]=a^{2}$ for every integer $r$ such that $1 \leq r \leq 5$ ). Hence, $\Delta_{H, D_{10}}^{g}$ is disconnected for each $g$ and each subgroup $H$ of order 2. Now, suppose that $|H|=5$. Then, we have $H=\left\{1, a, a^{2}, a^{3}, a^{4}\right\}$. In this case, the vertices $a, a^{2}, a^{3}$ and $a^{4}$ make a cycle in $\Delta_{H, D_{10}}^{g}$ for each $g$ as they commute among themselves.

If $G \cong D_{12}=\langle a, b| a^{6}=b^{2}=1$ and $\left.b a b^{-1}=a^{-1}\right\rangle$, then $g \in\left\{a^{2}, a^{4}\right\}$ and $|H|=$ $2,3,4,6$. We have $\Delta_{H, D_{12}}^{a^{2}}=\Delta_{H, D_{12}}^{a^{4}}$ since $\left(a^{2}\right)^{-1}=a^{4}$. Suppose that $|H|=2$, then $H=$ $Z\left(D_{12}\right)$ or $\left\langle a^{r} b\right\rangle$, for every integer $r$ such that $1 \leq r \leq 6$. For $H=Z\left(D_{12}\right), \Delta_{H, D_{12}}^{g}$ is an empty graph. For $H=\left\langle a^{r} b\right\rangle$, in each case, $a$ is an isolated vertex in $G \backslash H$ (since $\left[a, a^{r} b\right]=a^{2}$ for every integer $r$ such that $1 \leq r \leq 6)$ and hence $\Delta_{H, D_{12}}^{g}$ is disconnected. If $|H|=3$, then $H=\left\{1, a^{2}, a^{4}\right\}$. In this case, the vertices $a, a^{2}$ and $a^{4}$ make a triangle in $\Delta_{H, D_{12}}^{g}$ since they commute among themselves. If $|H|=4$, then $H=\left\{1, a^{3}, b, a^{3} b\right\},\left\{1, a^{3}, a b, a^{4} b\right\}$ or $\left\{1, a^{3}, a^{2} b, a^{5} b\right\}$. For all these $H, a$ is an isolated vertex in $G \backslash H$ (since $\left[a, a^{r} b\right]=a^{2}$ for every integer $r$ such that $1 \leq r \leq 6)$ and hence $\Delta_{H, D_{12}}^{g}$ is disconnected. If $|H|=6$, then $H=\left\{1, a, a^{2}, a^{3}, a^{4}, a^{5}\right\},\left\{1, a^{2}, a^{4}, b, a^{2} b, a^{4} b\right\}$ or $\left\{1, a^{2}, a^{4}, a b, a^{3} b, a^{5} b\right\}$. For all these $H$, the vertices $a, a^{2}, a^{4}$ and $a^{5}$ make a cycle in $\Delta_{H, D_{12}}^{g}$ since they commute among themselves.

If $G \cong Q_{12}=\langle a, b| a^{6}=1, b^{2}=a^{3}$ and $\left.b a b^{-1}=a^{-1}\right\rangle$, then $g \in\left\{a^{2}, a^{4}\right\}$ and $|H|=2,3,4,6$. We have $\Delta_{H, Q_{12}}^{a^{2}}=\Delta_{H, Q_{12}}^{a^{4}}$ since $\left(a^{2}\right)^{-1}=a^{4}$. If $|H|=2$, then $H=Z\left(Q_{12}\right)$ and so $\Delta_{H, Q_{12}}^{g}$ is an empty graph. If $|H|=3$, then $H=\left\{1, a^{2}, a^{4}\right\}$. In this case, the vertices $a, a^{2}$ and $a^{4}$ make a triangle in $\Delta_{H, Q_{12}}^{g}$ since they commute among themselves. If $|H|=4$, then $H=\left\{1, a^{3}, b, a^{3} b\right\},\left\{1, a^{3}, a b, a^{4} b\right\}$ or $\left\{1, a^{3}, a^{2} b, a^{5} b\right\}$. For all these $H, a$ is an isolated vertex in $G \backslash H$ (since $\left[a, a^{r} b\right]=a^{2}$ for every integer $r$ such that $1 \leq r \leq 6$ ) and hence $\Delta_{H, Q_{12}}^{g}$ is disconnected. If $|H|=6$, then $H=\left\{1, a, a^{2}, a^{3}, a^{4}, a^{5}\right\}$. In this case, the vertices $a, a^{2}, a^{4}$ and $a^{5}$ make a cycle in $\Delta_{H, Q_{12}}^{g}$ since they commute among themselves.

\section{Connectivity and Diameter}

Connectivity of $\Delta_{G}^{g}$ was studied in [19-21]. It was conjectured that the diameter of $\Delta_{G}^{g}$ is equal to 2 if $\Delta_{G}^{g}$ is connected. In this section, we discuss the connectivity of $\Delta_{H, G}^{g}$. In general, $\Delta_{H, G}^{g}$ is not connected. For any two vertices $x$ and $y$, we write $x \sim y$ and $x \nsim y$ respectively to mean that they are adjacent or not. We write $d(x, y)$ and $\operatorname{diam}\left(\Delta_{H, G}^{g}\right)$ to denote the distance between the vertices $x, y$ and diameter of $\Delta_{H, G}^{g}$, respectively.

Theorem 6. If $g \in H \backslash Z(G)$ and $g^{2}=1$, then $\operatorname{diam}\left(\Delta_{H, G}^{g}\right)=2$.

Proof. Let $x \neq g$ be any vertex of $\Delta_{H, G}^{g}$. Then, $[x, g] \neq g$ which implies $[x, g] \neq g^{-1}$ since $g^{2}=1$. Since $g \in H$, it follows that $x \sim g$. Therefore, $d(x, g)=2$ and hence $\operatorname{diam}\left(\Delta_{H, G}^{g}\right)=2$.

Lemma 1. Let $g \in H \backslash Z(H, G)$ such that $g^{2} \neq 1$ and $o(g) \neq 3$, where $o(g)$ is the order of $g$. If $x \in G \backslash Z(H, G)$ and $x \nsim g$, then $x \sim g^{2}$.

Proof. Since $g \neq 1$ and $x \nsim g$, it follows that $[x, g]=g^{-1}$. We have

$$
\left[x, g^{2}\right]=[x, g][x, g]^{g}=g^{-2} \neq g, g^{-1} .
$$

If $g^{2} \in Z(H, G)$, then, by (5), we have $g^{-2}=\left[x, g^{2}\right]=1$, a contradiction. Therefore, $g^{2} \in H \backslash Z(H, G)$. Hence, $x \sim g^{2}$.

Theorem 7. Let $g \in H \backslash Z(H, G)$ and $o(g) \neq 3$. Then, $\operatorname{diam}\left(\Delta_{H, G}^{g}\right) \leq 3$. 
Proof. If $g^{2}=1$, then, by Theorem 6 , we have $\operatorname{diam}\left(\Delta_{H, G}^{g}\right)=2$. Therefore, we assume that $g^{2} \neq 1$. Let $x, y$ be any two vertices of $\Delta_{H, G}^{g}$ such that $x \nsim y$. Therefore, $[x, y]=g$ or $g^{-1}$. If $x \sim g$ and $y \sim g$, then $x \sim g \sim y$ and so $d(x, y)=2$. If $x \nsim g$ and $y \nsim g$, then, by Lemma 1, we have $x \sim g^{2} \sim y$ and so $d(x, y)=2$. Therefore, we shall not consider these two situations in the following cases.

Case 1: $x, y \in H$

Suppose that one of $x, y$ is adjacent to $g$ and the other is not. Without any loss, we assume that $x \nsim g$ and $y \sim g$. Then, $[x, g]=g^{-1}$ and $[y, g] \neq g, g^{-1}$. By Lemma 1 , we have $x \sim g^{2}$.

Consider the element $y g \in H$. If $y g \in Z(H, G)$, then $\left[y, g^{2}\right]=1 \neq g, g^{-1}$. Therefore, $x \sim g^{2} \sim y$ and so $d(x, y)=2$.

If $y g \notin Z(H, G)$, then we have $[x, y g]=[x, g][x, y]^{g}=g^{-1}[x, y]^{g} \neq g, g^{-1}$. In addition, $[y, y g]=[y, g] \neq g, g^{-1}$. Hence, $x \sim y g \sim y$ and so $d(x, y)=2$.

Case 2: One of $x, y$ belongs to $H$ and the other does not.

Without any loss, assume that $x \in H$ and $y \notin H$. If $x \nsim g$ and $y \sim g$, then, by Lemma 1, we have $x \sim g^{2}$. In addition, $\left[g, g^{2}\right]=1 \neq g, g^{-1}$ and so $g^{2} \sim g$. Therefore, $x \sim g^{2} \sim g \sim y$ and hence $d(x, y) \leq 3$. If $x \sim g$ and $y \nsim g$, then $[x, g] \neq g, g^{-1}$ and $[y, g]=g^{-1}$. By Lemma 1 , we have $y \sim g^{2}$. Consider the element $x g \in H$. If $x g \in Z(H, G)$, then $\left[x, g^{2}\right]=1 \neq g, g^{-1}$. Therefore, $x \sim g^{2}$ and so $y \sim g^{2} \sim x$. Thus, $d(x, y)=2$.

If $x g \notin Z(H, G)$, then we have $[y, x g]=[y, g][y, x]^{g}=g^{-1}[y, x]^{g} \neq g, g^{-1}$. In addition, $[x, x g]=[x, g] \neq g, g^{-1}$. Hence, $y \sim x g \sim x$ and so $d(x, y)=2$.

Case 3: $x, y \notin H$.

Suppose that one of $x, y$ is adjacent to $g$ and the other is not. Without any loss, we assume that $x \nsim g$ and $y \sim g$. Then, by Lemma 1, we have $x \sim g^{2}$. In addition, $\left[g, g^{2}\right]=1 \neq g, g^{-1}$ and so $g^{2} \sim g$. Therefore, $x \sim g^{2} \sim g \sim y$ and hence $d(x, y) \leq 3$.

Thus, $d(x, y) \leq 3$ for all $x, y \in G \backslash Z(H, G)$. Hence, the result follows.

The rest part of this paper is devoted to the study of connectivity of $\Delta_{H, D_{2 n}}^{g}$, where $D_{2 n}=\left\langle a, b: a^{n}=b^{2}=1, b a b^{-1}=a^{-1}\right\rangle$ is the dihedral group of order $2 n$. It is well known that $Z\left(D_{2 n}\right)=\{1\}$, the commutator subgroup $D_{2 n}^{\prime}=\langle a\rangle$ if $n$ is odd and $Z\left(D_{2 n}\right)=\left\{1, a^{\frac{n}{2}}\right\}$ and $D_{2 n}^{\prime}=\left\langle a^{2}\right\rangle$ if $n$ is even. By (Theorem 4, [19]), it follows that $\Delta_{D_{2 n}}^{g}$ is disconnected if $n=3,4,6$. Therefore, we consider $n \geq 8$ and $n \geq 5$ accordingly, as $n$ is even or odd in the following results.

Theorem 8. Consider the graph $\Delta_{H, D_{2 n}}^{g}$ where $n(\geq 8)$ is even. If $H=\langle a\rangle$, then $\Delta_{H, D_{2 n}}^{g}$ is connected and $\operatorname{diam}\left(\Delta_{H, D_{2 n}}^{g}\right)=2$.

(b) Let $H=\left\langle a^{\frac{n}{2}}, a^{r} b\right\rangle$ for $0 \leq r<\frac{n}{2}$. Then, $\Delta_{H, D_{2 n}}^{g}$ is connected with diameter 2 if $g=1$ and $\Delta_{H, D_{2 n}}^{g}$ is not connected if $g \neq 1$.

(c) If $H=\left\langle a^{r} b\right\rangle$ for $1 \leq r \leq n$, then $\Delta_{H, D_{2 n}}^{g}$ is not connected.

Proof. Since $n$ is even, we have $g=a^{2 i}$ for $1 \leq i \leq \frac{n}{2}$.

(a) Case 1: $g=1$

Since $H$ is abelian, the induced subgraph of $\Delta_{H, D_{2 n}}^{g}$ on $H \backslash Z\left(H, D_{2 n}\right)$ is empty. Thus, we need to see the adjacency of these vertices with those in $D_{2 n} \backslash H$. Suppose that $\left[a^{r} b, a^{j}\right]=$ 1 and $\left[b, a^{j}\right]=1$ for every integer $r, j$ such that $1 \leq r, j \leq n-1$. Then, $a^{2 j}=a^{0}$ or $a^{n}$ and so $j=0$ or $j=\frac{n}{2}$. Therefore, every vertex in $H \backslash Z\left(H, D_{2 n}\right)$ is adjacent to all the vertices in $D_{2 n} \backslash H$. Thus, $\Delta_{H, D_{2 n}}^{g}$ is connected and $\operatorname{diam}\left(\Delta_{H, D_{2 n}}^{g}\right)=2$.

Case 2: $g \neq 1$

Since $H$ is abelian, the induced subgraph of $\Delta_{H, D_{2 n}}^{g}$ on $H \backslash Z\left(H, D_{2 n}\right)$ is a complete graph. Therefore, it is sufficient to prove that no vertex in $D_{2 n} \backslash H$ is isolated. If $g \neq g^{-1}$, then $g \neq a^{\frac{n}{2}}$. Suppose that $\left[a^{r} b, a^{j}\right]=g$ and $\left[b, a^{j}\right]=g$ for every integer $r, j$ such that 
$1 \leq r, j \leq n-1$. Then, $a^{2 j}=a^{2 i}$ and so $j=i$ or $j=\frac{n}{2}+i$. If $\left[a^{r} b, a^{j}\right]=g^{-1}$ and $\left[b, a^{j}\right]=g^{-1}$ for every integer $r, j$ such that $1 \leq r, j \leq n-1$, then $a^{2 j}=a^{n-2 i}$ and so $j=n-i$ or $j=\frac{n}{2}-i$. Therefore, there exists an integer $j$ such that $1 \leq j \leq n-1$ and $j \neq i, \frac{n}{2}+i, n-i$ and $\frac{n}{2}-i$ for which $a^{j}$ is adjacent to all the vertices in $D_{2 n} \backslash H$. If $g=g^{-1}$, then $g=a^{\frac{n}{2}}$. Suppose that $\left[a^{r} b, a^{j}\right]=g$ and $\left[b, a^{j}\right]=g$ for every integer $r, j$ such that $1 \leq r, j \leq n-1$, then $a^{2 j}=a^{\frac{n}{2}}$ and so $j=\frac{n}{4}$ or $j=\frac{3 n}{4}$. Therefore, there exists an integer $j$ such that $1 \leq j \leq n-1$ and $j \neq \frac{n}{4}$ and $\frac{3 n}{4}$ for which $a^{j}$ is adjacent to all the vertices in $D_{2 n} \backslash H$. Thus, $\Delta_{H, D_{2 n}}^{g}$ is connected and $\operatorname{diam}\left(\Delta_{H, D_{2 n}}^{g}\right)=2$.

(b) Case 1: $g=1$

We have $\left[a^{\frac{n}{2}+r} b, a^{r} b\right]=1$ for every integer $r$ such that $1 \leq r \leq n$. Therefore, the induced subgraph of $\Delta_{H, D_{2 n}}^{g}$ on $H \backslash Z\left(H, D_{2 n}\right)$ is empty. Thus, we need to see the adjacency of these vertices with those in $D_{2 n} \backslash H$. Suppose $\left[a^{r} b, a^{i}\right]=1$ and $\left[a^{\frac{n}{2}+r} b, a^{i}\right]=1$ for every integer $i$ such that $1 \leq i \leq n-1$. Then, $a^{2 i}=a^{n}$ and so $i=\frac{n}{2}$. Therefore, for every integer $i$ such that $1 \leq i \leq n-1$ and $i \neq \frac{n}{2}, a^{i}$ is adjacent to both $a^{r} b$ and $a^{\frac{n}{2}+r} b$. In addition, we have $\left[a^{s} b, a^{r} b\right]=a^{2(s-r)}$ and $\left[a^{\frac{n}{2}+r} b, a^{s} b\right]=a^{2\left(\frac{n}{2}+r-s\right)}$ for every integer $s$ such that $1 \leq s \leq n$. Suppose $\left[a^{s} b, a^{r} b\right]=1$ and $\left[a^{\frac{n}{2}+r} b, a^{s} b\right]=1$. Then, $s=r$ or $s=\frac{n}{2}+r$. Therefore, for every integer $s$ such that $1 \leq s \leq n$ and $s \neq r, \frac{n}{2}+r, a^{s} b$ is adjacent to both $a^{r} b$ and $a^{\frac{n}{2}+r} b$. Thus, $\Delta_{H, D_{2 n}}^{g}$ is connected and $\operatorname{diam}\left(\Delta_{H, D_{2 n}}^{g}\right)=2$.

Case 2: $g \neq 1$

If $H=\left\langle a^{\frac{n}{2}}, a^{r} b\right\rangle=\left\{1, a^{\frac{n}{2}}, a^{r} b, a^{\frac{n}{2}+r} b\right\}$ for $0 \leq r<\frac{n}{2}$, then $H \backslash Z\left(H, D_{2 n}\right)=\left\{a^{r} b, a^{\frac{n}{2}+r} b\right\}$. We have $\left[a^{r} b, a^{i}\right]=a^{2 i}=\left[a^{\frac{n}{2}+r} b, a^{i}\right]$ for every integer $i$ such that $1 \leq i \leq \frac{n}{2}-1$. That is, $\left[a^{r} b, a^{i}\right]=g$ and $\left[a^{\frac{n}{2}+r} b, a^{i}\right]=g$ for every integer $i$ such that $1 \leq i \leq \frac{n}{2}-1$. Thus, $a^{i}$ is an isolated vertex in $D_{2 n} \backslash H$. Hence, $\Delta_{H, D_{2 n}}^{g}$ is not connected.

(c) Case 1: $g=1$

We have $\left[a^{\frac{n}{2}+r} b, a^{r} b\right]=1$ for every integer $r$ such that $1 \leq r \leq n$. Thus, $a^{\frac{n}{2}+r} b$ is an isolated vertex in $D_{2 n} \backslash H$. Hence, $\Delta_{H, D_{2 n}}^{g}$ is not connected.

Case 2: $g \neq 1$

If $H=\left\langle a^{r} b\right\rangle=\left\{1, a^{r} b\right\}$ for $1 \leq r \leq n$, then $H \backslash Z\left(H, D_{2 n}\right)=\left\{a^{r} b\right\}$. We have $\left[a^{r} b, a^{i}\right]=a^{2 i}=g$ for every integer $i$ such that $1 \leq i \leq \frac{n}{2}-1$. Thus, $a^{i}$ is an isolated vertex in $D_{2 n} \backslash H$. Hence, $\Delta_{H, D_{2 n}}^{g}$ is not connected.

Theorem 9. Consider the graph $\Delta_{H, D_{2 n}}^{g}$, where $n(\geq 8)$ and $\frac{n}{2}$ are even.

(a) If $H=\left\langle a^{2}\right\rangle$, then $\Delta_{H, D_{2 n}}^{g}$ is connected with diameter 2 if and only if $g \notin\left\langle a^{4}\right\rangle$.

(b) If $H=\left\langle a^{2}, b\right\rangle$ or $\left\langle a^{2}, a b\right\rangle$, then $\Delta_{H, D_{2 n}}^{g}$ is connected and $\operatorname{diam}\left(\Delta_{H, D_{2 n}}^{g}\right) \leq 3$.

Proof. Since $n$ is even, we have $g=a^{2 i}$ for $1 \leq i \leq \frac{n}{2}$.

(a) Case 1: $g=1$

We know that the vertices in $H$ commutes with all the odd powers of $a$. That is, any vertex in $\Delta_{H, D_{2 n}}^{g}$ of the form $a^{i}$, where $i$ is an odd integer and $1 \leq i \leq n-1$, is not adjacent with any vertex. Hence, $\Delta_{H, D_{2 n}}^{g}$ is not connected.

Case 2: $g \neq 1$

Since $H$ is abelian, the induced subgraph of $\Delta_{H, D_{2 n}}^{g}$ on $H \backslash Z\left(H, D_{2 n}\right)$ is a complete graph. In addition, the vertices in $H$ commutes with all the odd powers of $a$. That is, a vertex of the form $a^{i}$, where $i$ is an odd integer, in $\Delta_{H, D_{2 n}}^{g}$ is adjacent with all the vertices in $H$. We have $\left[a^{r} b, a^{2 i}\right]=a^{4 i}$ and $\left[b, a^{2 i}\right]=a^{4 i}$ for every integer $r, i$ such that $1 \leq r \leq n-1$ and $1 \leq i \leq \frac{n}{2}-1$. Thus, for $g \notin\left\langle a^{4}\right\rangle$, every vertex of $H$ is adjacent to the vertices of the form $a^{r} b$, where $1 \leq r \leq n$. Therefore, $\Delta_{H, D_{2 n}}^{g}$ is connected and $\operatorname{diam}\left(\Delta_{H, D_{2 n}}^{g}\right)=2$. In addition, if $g=a^{4 i}$ for some integer $i$ where $1 \leq i \leq \frac{n}{4}-1$ (i,e., $g \in\left\langle a^{4}\right\rangle$ ), then the vertices $a^{r} b \in D_{2 n} \backslash H$, where $1 \leq r \leq n$, will remain isolated. Hence, $\Delta_{H, D_{2 n}}^{g}$ is disconnected in this case. This completes the proof of part (a). 
(b) Case 1: $g=1$

Suppose that $H=\left\langle a^{2}, b\right\rangle$. Then, $a^{2 i} \nsim a^{j}$ but $a^{2 i} \sim a^{r} b$ for all $i, j, r$ such that $1 \leq i \leq$ $\frac{n}{2}-1, i \neq \frac{n}{4} ; 1 \leq j \leq n-1$ is an odd number and $1 \leq r \leq n$ because $\left[a^{2 i}, a^{j}\right]=1$ and $\left[a^{2 i}, a^{r} b\right]=a^{4 i}$. We shall find a path to $a^{j}$, where $1 \leq j \leq n-1$ is an odd number. We have $\left[a^{j}, b\right]=a^{2 j} \neq 1$ and $a^{j} \in G \backslash H$ for all $j$ such that $1 \leq j \leq n-1$ is an odd number. Therefore, $a^{2 i} \sim b \sim a^{j}$. Hence, $\Delta_{H, D_{2 n}}^{g}$ is connected and diam $\left(\Delta_{H, D_{2 n}}^{g}\right) \leq 3$.

If $H=\left\langle a^{2}, a b\right\rangle$, then $a^{2 i} \nsim a^{j}$ but $a^{2 i} \sim a^{r} b$ for all $i, j, r$ such that $1 \leq i \leq \frac{n}{2}-1, i \neq \frac{n}{4}$; $1 \leq j \leq n-1$ is an odd number and $1 \leq r \leq n$ because $\left[a^{2 i}, a^{j}\right]=1$ and $\left[a^{2 i}, a^{r} b\right]=a^{4 i}$. We shall find a path to $a^{j}$, where $1 \leq j \leq n-1$ is an odd number. We have $\left[a^{j}, a b\right]=a^{2 j} \neq 1$ and $a^{j} \in G \backslash H$ for all $j$ such that $1 \leq j \leq n-1$ is an odd number. Therefore, $a^{2 i} \sim a b \sim a^{j}$. Hence, $\Delta_{H, D_{2 n}}^{g}$ is connected and $\operatorname{diam}\left(\Delta_{H, D_{2 n}}^{g}\right) \leq 3$.

Case 2: $g \neq 1$

We have $\left\langle a^{2}\right\rangle \subset H$. Therefore, if $g \notin\left\langle a^{4}\right\rangle$, then every vertex in $\left\langle a^{2}\right\rangle$ is adjacent to all other vertices in both cases (as discussed in part (a)). Hence, $\Delta_{H, D_{2 n}}^{g}$ is connected and $\operatorname{diam}\left(\Delta_{H, D_{2 n}}^{g}\right)=2$. Suppose that $g=a^{4 i}$ for some integer $i$, where $1 \leq i \leq \frac{n}{4}-1$.

Suppose that $H=\left\langle a^{2}, b\right\rangle$. Then, $a^{2 i} \sim a^{j}$ but $a^{2 i} \nsim a^{r} b$ for all $j, r$ such that $1 \leq j \leq n-1$ is an odd number and $1 \leq r \leq n$ because $\left[a^{2 i}, a^{j}\right]=1$ and $\left[a^{2 i}, a^{r} b\right]=a^{4 i}$. We shall find a path between $a^{2 i}$ and $a^{r} b$ for all $i, r$ such that $1 \leq i \leq \frac{n}{2}-1$ and $1 \leq r \leq n$. We have $\left[a^{j}, b\right]=a^{2 j} \neq a^{4 i}$ and $a^{j} \in G \backslash H$ for all $j$ such that $1 \leq j \leq n-1$ is an odd number. Therefore, $a^{2 i} \sim a^{j} \sim b$. Consider the vertices of the form $a^{r} b$, where $1 \leq r \leq n-1$. We have $\left[a^{r} b, b\right]=a^{2 r}$. Suppose $\left[a^{r} b, b\right]=g$, then it gives $a^{2 r}=a^{4 i}$, which implies $r=2 i$ or $r=\frac{n}{2}+2 i$. Therefore, $b \sim a^{r} b$ if and only if $r \neq 2 i$ and $r \neq \frac{n}{2}+2 i$. Thus, we have $a^{2 i} \sim a^{j} \sim b \sim a^{r} b$, where $1 \leq r \leq n-1$ and $r \neq 2 i$ and $r \neq \frac{n}{2}+2 i$. Again, we know that $a^{\frac{n}{2}+2 i} b, a^{2 i} b \in H$ and $\left[a^{\frac{n}{2}+2 i} b, a^{2 i} b\right]=1$, so $a^{\frac{n}{2}+2 i} b \sim a^{2 i} b$. If we are able to find a path between $a^{j}$ and any one of $a^{\frac{n}{2}+2 i} b$ and $a^{2 i} b$, then we are done. Now, $\left[a^{2 i} b, a^{j}\right] \neq a^{4 i}$ and $\left[a^{\frac{n}{2}+2 i} b, a^{j}\right] \neq a^{4 i}$ for any odd number $j$ such that $1 \leq j \leq n-1$ so we have $a^{\frac{n}{2}+2 i} b \sim a^{j} \sim a^{2 i} b$. Thus, $a^{2 i} \sim a^{j} \sim a^{2 i} b, a^{2 i} \sim a^{j} \sim a^{\frac{n}{2}+2 i} b, a^{r} b \sim b \sim a^{j} \sim a^{2 i} b$ and $a^{r} b \sim b \sim a^{j} \sim a^{\frac{n}{2}+2 i} b$, where $1 \leq r \leq n-1$ and $r \neq 2 i$ and $r \neq \frac{n}{2}+2 i$. Hence, $\Delta_{H, D_{2 n}}^{g}$ is connected and $\operatorname{diam}\left(\Delta_{H, D_{2 n}}^{g}\right) \leq 3$.

If $H=\left\langle a^{2}, a b\right\rangle$, then $a^{2 i} \sim a^{j}$ but $a^{2 i} \nsim a^{r} b$ for all $j, r$ such that $1 \leq j \leq n-1$ is an odd number and $1 \leq r \leq n$ because $\left[a^{2 i}, a^{j}\right]=1$ and $\left[a^{2 i}, a^{r} b\right]=a^{4 i}$. We shall find a path between $a^{2 i}$ and $a^{r} b$ for all $i, r$ such that $1 \leq i \leq \frac{n}{2}-1$ and $1 \leq r \leq n$. We have $\left[a^{j}, a b\right]=a^{2 j} \neq a^{4 i}$ and $a^{j} \in G \backslash H$ for all $j$ such that $1 \leq j \leq n-1$ is an odd number. Thus, we have $a^{2 i} \sim a^{j} \sim a b$. Consider the vertices of the form $a^{r} b$, where $2 \leq r \leq n$. We have $\left[a^{r} b, a b\right]=a^{2(r-1)}$. Suppose $\left[a^{r} b, a b\right]=g$, then it gives $a^{2(r-1)}=a^{4 i}$ which implies $r=2 i+1$ or $r=\frac{n}{2}+2 i+1$. Therefore, $a b \sim a^{r} b$ if and only if $r \neq 2 i+1$ and $r \neq \frac{n}{2}+2 i+1$. Thus, we have $a^{2 i} \sim a^{j} \sim a b \sim a^{r} b$, where $2 \leq r \leq n$ and $r \neq 2 i+1$ and $r \neq \frac{n}{2}+2 i+1$. Again, we know that $a^{\frac{n}{2}+2 i+1} b, a^{2 i+1} b \in H$ and $\left[a^{\frac{n}{2}+2 i+1} b, a^{2 i+1} b\right]=1$, so $a^{\frac{n}{2}+2 i+1} b \sim a^{2 i+1} b$. If we are able to find a path between $a^{j}$ and any one of $a^{\frac{n}{2}+2 i+1} b$ and $a^{2 i+1} b$, then we are done. Now, $\left[a^{2 i+1} b, a^{j}\right] \neq a^{4 i}$ and $\left[a^{\frac{n}{2}+2 i+1} b, a^{j}\right] \neq a^{4 i}$ for any odd number $j$ such that $1 \leq j \leq n-1$ so we have $a^{\frac{n}{2}+2 i+1} b \sim a^{j} \sim a^{2 i+1} b$. Thus, $a^{2 i} \sim a^{j} \sim a^{2 i+1} b, a^{2 i} \sim a^{j} \sim a^{\frac{n}{2}+2 i+1} b$, $a^{r} b \sim a b \sim a^{j} \sim a^{2 i+1} b$ and $a^{r} b \sim a b \sim a^{j} \sim a^{\frac{n}{2}+2 i+1} b$, where $2 \leq r \leq n$ and $r \neq 2 i+1$ and $r \neq \frac{n}{2}+2 i+1$. Hence, $\Delta_{H, D_{2 n}}^{g}$ is connected and $\operatorname{diam}\left(\Delta_{H, D_{2 n}}^{g}\right) \leq 3$.

Theorem 10. Consider the graph $\Delta_{H, D_{2 n}}^{g}$, where $n(\geq 8)$ is even and $\frac{n}{2}$ is odd.

(a) If $H=\left\langle a^{2}\right\rangle$, then $\Delta_{H, D_{2 n}}^{g}$ is not connected if $g=1$ and $\Delta_{H, D_{2 n}}^{g}$ is connected with $\operatorname{diam}\left(\Delta_{H, D_{2 n}}^{g}\right)=2$ if $g \neq 1$.

(b) If $H=\left\langle a^{2}, b\right\rangle$ or $\left\langle a^{2}, a b\right\rangle$, then $\Delta_{H, D_{2 n}}^{g}$ is not connected if $g=1$ and $\Delta_{H, D_{2 n}}^{g}$ is connected with $\operatorname{diam}\left(\Delta_{H, D_{2 n}}^{g}\right)=2$ if $g \neq 1$. 
Proof. Since $n$ is even, we have $g=a^{2 i}$ for $1 \leq i \leq \frac{n}{2}$.

(a) Case 1: $g=1$

We know that the vertices in $H$ commute with all the odd powers of $a$. That is, any vertex of the form $a^{i} \in D_{2 n} \backslash H$, where $i$ is an odd integer, is not adjacent with any vertex in $\Delta_{H, D_{2 n}}^{g}$. Hence, $\Delta_{H, D_{2 n}}^{g}$ is not connected.

Case 2: $g \neq 1$

Since $H$ is abelian, the induced subgraph of $\Delta_{H, D_{2 n}}^{g}$ on $H \backslash Z\left(H, D_{2 n}\right)$ is a complete graph. In addition, the vertices in $H$ commute with all the odd powers of $a$. That is, a vertex of the form $a^{i}$, where $i$ is an odd integer, in $\Delta_{H, D_{2 n}}^{g}$ is adjacent with all the vertices in $H$. We claim that at least one element of $H \backslash Z\left(H, D_{2 n}\right)$ is adjacent to all $a^{r} b^{\prime}$ s such that $1 \leq r \leq n$. Consider the following cases.

Subcase 1: $g^{3} \neq 1$

If $\left[g, a^{r} b\right]=g$, i.e., $\left[a^{2 i}, a^{r} b\right]=a^{2 i}$ for all $1 \leq i \leq \frac{n}{2}-1$ and $1 \leq r \leq n$, then we get $g=a^{2 i}=1$, a contradiction. If $\left[g, a^{r} b\right]=g^{-1}$, i.e., $\left[a^{2 i}, a^{r} b\right]=a^{n-2 i}$ for all $1 \leq i \leq \frac{n}{2}-1$ and $1 \leq r \leq n$, then we get $g^{3}=\left(a^{2 i}\right)^{3}=a^{6 i}=1$, a contradiction. Therefore, $g$ is adjacent to all other vertices of the form $a^{r} b$ such that $1 \leq r \leq n$.

Subcase 2: $g^{3}=1$

If $\left[g, a^{r} b\right]=g^{-1}$, i.e., $\left[a^{2 i}, a^{r} b\right]=a^{2 i}$, then $\left[g a^{2}, a^{r} b\right]=g^{-1} a^{4}$ for all $1 \leq i \leq \frac{n}{2}-1$ and $1 \leq r \leq n$. Now, if $g^{-1} a^{4}=g^{-1}$, then $a^{4}=1$, a contradiction since $a^{n}=1$ for $n \geq 8$. If $g^{-1} a^{4}=g$, then $a^{n-2 i-4}=1$ for all $1 \leq i \leq \frac{n}{2}-1$, which is a contradiction since $1 \leq i \leq \frac{n}{2}-1$. Therefore, $g a^{2}$ is adjacent to all other vertices of the form $a^{r} b$ such that $1 \leq r \leq n$.

Thus, there exists a vertex in $H \backslash Z\left(H, D_{2 n}\right)$, which is adjacent to all other vertices in $D_{2 n}$. Hence, $\Delta_{H, D_{2 n}}^{g}$ is connected and $\operatorname{diam}\left(\Delta_{H, D_{2 n}}^{g}\right)=2$.

(b) Case 1: $g=1$

We know that the vertices in $H$ commute with the vertex $a^{\frac{n}{2}}$. That is, the vertex $a^{\frac{n}{2}} \in D_{2 n} \backslash H$ is not adjacent with any vertex in $\Delta_{H, D_{2 n}}^{g}$. Hence, $\Delta_{H, D_{2 n}}^{g}$ is not connected.

Case 2: $g \neq 1$

As shown in Case 2 of part (a), it can be seen that either $g$ or $g a^{2}$ is adjacent to all other vertices. Hence, $\Delta_{H, D_{2 n}}^{g}$ is connected and $\operatorname{diam}\left(\Delta_{H, D_{2 n}}^{g}\right)=2$.

Theorem 11. Consider the graph $\Delta_{H, D_{2 n}}^{g}$, where $n(\geq 5)$ is odd.

(a) If $H=\langle a\rangle$, then $\Delta_{H, D_{2 n}}^{g}$ is connected and $\operatorname{diam}\left(\Delta_{H, D_{2 n}}^{g}\right)=2$.

(b) If $H=\left\langle a^{r} b\right\rangle$, where $1 \leq r \leq n$, then $\Delta_{H, D_{2 n}}^{g}$ is connected with $\operatorname{diam}\left(\Delta_{H, D_{2 n}}^{g}\right)=2$ if $g=1$ and $\Delta_{H, D_{2 n}}^{g}$ is not connected if $g \neq 1$.

Proof. Since $n$ is odd, we have $g=a^{i}$ for $1 \leq i \leq n$.

(a) Case 1: $g=1$

Since $H$ is abelian, the induced subgraph of $\Delta_{H, D_{2 n}}^{g}$ on $H \backslash Z\left(H, D_{2 n}\right)$ is empty. Therefore, we need to see the adjacency of these vertices with those in $D_{2 n} \backslash H$. Suppose that $\left[a^{r} b, a^{j}\right]=1$ and $\left[b, a^{j}\right]=1$ for every integer $r, j$ such that $1 \leq r, j \leq n-1$. Then, $a^{2 j}=a^{n}$ and so $j=\frac{n}{2}$, a contradiction. Therefore, for every integer $j$ such that $1 \leq j \leq n-1, a^{j}$ is adjacent to all the vertices in $D_{2 n} \backslash H$. Thus, $\Delta_{H, D_{2 n}}^{g}$ is connected and $\operatorname{diam}\left(\Delta_{H, D_{2 n}}^{g}\right)=2$.

Case 2: $g \neq 1$

Since $H$ is abelian, the induced subgraph of $\Delta_{H, D_{2 n}}^{g}$ on $H \backslash Z\left(H, D_{2 n}\right)$ is a complete graph. Therefore, it is sufficient to prove that no vertex in $D_{2 n} \backslash H$ is isolated. Since $n$ is odd, we have $g \neq g^{-1}$. If $\left[a^{r} b, a^{j}\right]=g$ and $\left[b, a^{j}\right]=g$ for every integer $r, j$ such that $1 \leq r, j \leq n-1$, then $j=\frac{i}{2}$ or $j=\frac{n+i}{2}$. If $\left[a^{r} b, a^{j}\right]=g^{-1}$ and $\left[b, a^{j}\right]=g^{-1}$ for every integer $r, j$ such that $1 \leq r, j \leq n-1$, then $j=\frac{n-i}{2}$ or $j=n-\frac{i}{2}$. Therefore, there exists an integer $j$ such that $1 \leq j \leq n-1$ and $j \neq \frac{i}{2}, \frac{n+i}{2}, \frac{n-i}{2}$ and $n-\frac{i}{2}$ for which $a^{j}$ is adjacent to all other vertices in $D_{2 n} \backslash H$. Thus, $\Delta_{H, D_{2 n}}^{g}$ is connected and $\operatorname{diam}\left(\Delta_{H, D_{2 n}}^{g}\right)=2$. 
(b) Case 1: $g=1$

We have $\left[a^{r} b, a^{j}\right] \neq 1$ and $\left[b, a^{j}\right] \neq 1$ for every integer $r, j$ such that $1 \leq r, j \leq n-1$. Thus, $a^{r} b$ is adjacent to $a^{j}$ for every integer $j$ such that $1 \leq j \leq n-1$. In addition, we have $\left[a^{s} b, a^{r} b\right]=a^{2(s-r)}$ for every integer $r, s$ such that $1 \leq r, s \leq n$. Supposing that $\left[a^{s} b, a^{r} b\right]=1$, then $s=r$ as $s=\frac{n}{2}+r$ is not possible. Therefore, for every integer $r, s$ such that $1 \leq r, s \leq n$ and $s \neq r, a^{s} b$ is adjacent to $a^{r} b$. Thus, $\Delta_{H, D_{2 n}}^{g}$ is connected and $\operatorname{diam}\left(\Delta_{H, D_{2 n}}^{g}\right)=2$.

Case 2: $g \neq 1$

If $i$ is even, then $\left[a^{\frac{i}{2}}, a^{r} b\right]=a^{i}=g$ and so the vertex $a^{\frac{i}{2}}$ remains isolated. If $i$ is odd, then $n-i$ is even and we have $\left[a^{\frac{n-i}{2}}, a^{r} b\right]=a^{n-i}=g^{-1}$. Therefore, the vertex $a^{\frac{n-i}{2}}$ remains isolated. Hence, $\Delta_{H, D_{2 n}}^{g}$ is not connected.

Theorem 12. Consider the graph $\Delta_{H, D_{2 n}{ }^{\prime}}^{g}$ where $n(\geq 5)$ is odd.

(a) If $H=\left\langle a^{d}\right\rangle$, where $d \mid n$ and $o\left(a^{d}\right)=3$, then $\Delta_{H, D_{2 n}}^{g}$ is not connected.

(b) If $H=\left\langle a^{d}, b\right\rangle,\left\langle a^{d}, a b\right\rangle$ or $\left\langle a^{d}, a^{2} b\right\rangle$, where $d \mid n$ and $o\left(a^{d}\right)=3$, then $\Delta_{H, D_{2 n}}^{g}$ is connected with diameter 2 if $g \neq 1, a^{d}, a^{2 d}$.

(c) If $H=\left\langle a^{d}, b\right\rangle$, where $d \mid n$ and $o\left(a^{d}\right)=3$, then $\Delta_{H, D_{2 n}}^{g}$ is connected and $\operatorname{diam}\left(\Delta_{H, D_{2 n}}^{g}\right)=$ $\begin{cases}2, & \text { if } g=1 \\ 3, & \text { if } g=a^{d} \text { or } a^{2 d} .\end{cases}$

Proof. (a) Given $H=\left\{1, a^{d}, a^{2 d}\right\}$. We have $\left[a^{d}, a^{2 d}\right]=1,\left[a^{d}, a^{r} b\right]=a^{2 d}$ and $\left[a^{2 d}, a^{r} b\right]=$ $a^{4 d}=a^{d}$ for all $r$ such that $1 \leq r \leq n$. Therefore, $g=1$, $a^{d}$ or $a^{2 d}$. If $g=a^{d}$ or $a^{2 d}$, then $a^{d} \nsim a^{r} b$ and $a^{2 d} \nsim a^{r} b$ for all $r$ such that $1 \leq r \leq n$. Thus, $\Delta_{H, D_{2 n}}^{g}$ is disconnected. If $g=1$, then the vertex $a \in D_{2 n} \backslash H$ remains isolated because $\left[a^{d}, a\right]=1=\left[a^{2 d}, a\right]$. Hence, $\Delta_{H, D_{2 n}}^{g}$ is not connected.

(b) If $g \neq 1, a^{d}, a^{2 d}$, then $a^{d}$ is adjacent to all other vertices, as discussed in part (a). Hence, $\Delta_{H, D_{2 n}}^{g}$ is connected and $\operatorname{diam}\left(\Delta_{H, D_{2 n}}^{g}\right)=2$.

(c) Case 1: $g=1$

Since $n$ is odd, we have $2 i \neq n$ for all integers $i$ such that $1 \leq i \leq n-1$. Therefore, if $g=1$, then $b$ is adjacent to all other vertices because $\left[a^{i}, b\right]=a^{2 i}$ and $\left[a^{r} b, b\right]=a^{2 r}$ for all integers $i, r$ such that $1 \leq i, r \leq n-1$. Hence, $\Delta_{H, D_{2 n}}^{g}$ is connected and $\operatorname{diam}\left(\Delta_{H, D_{2 n}}^{g}\right)=2$.

Case 2: $g=a^{d}$ or $a^{2 d}$

Since $\left[a^{d}, a^{2 d}\right]=1$, we have $a^{d} \sim a^{2 d}$. In addition, all the vertices of the form $a^{i}$ commute among themselves, where $1 \leq i \leq n-1$. Therefore, $a^{d} \sim a^{i} \sim a^{2 d}$ for all $1 \leq i \leq n-1$ such that $i \neq d, 2 d$. Again, $\left[a^{i}, a^{r} b\right]=a^{2 i}=\left[a^{i}, b\right]$ for all $1 \leq i, r \leq n-1$. If $\left[a^{i}, a^{r} b\right]=a^{d}$ or $a^{2 d}$ for all $1 \leq r \leq n$, then $i=2 d$ or $d$ respectively. Therefore, $a^{d} \sim a^{i} \sim b$, $a^{d} \sim a^{i} \sim a^{d} b, a^{d} \sim a^{i} \sim a^{2 d} b, a^{2 d} \sim a^{i} \sim b, a^{2 d} \sim a^{i} \sim a^{d} b$ and $a^{2 d} \sim a^{i} \sim a^{2 d} b$ for all $1 \leq i \leq n-1$ such that $i \neq d, 2 d$. If $\left[a^{r} b, b\right]=a^{d}$ or $a^{2 d}$ for all $1 \leq r \leq n-1$, then $a^{2 r}=a^{d}$ or $a^{2 d}$, which gives $r=2 d$ or $d$, respectively. Therefore, $a^{d} \sim a^{i} \sim b \sim a^{r} b, a^{2 d} \sim a^{i} \sim b \sim a^{r} b$, $a^{d} b \sim a^{i} \sim b \sim a^{r} b$ and $a^{2 d} b \sim a^{i} \sim b \sim a^{r} b$ for all $1 \leq i, r \leq n-1$ such that $i, r \neq d, 2 d$. Hence, $\Delta_{H, D_{2 n}}^{g}$ is connected and $\operatorname{diam}\left(\Delta_{H, D_{2 n}}^{g}\right)=3$.

\section{Conclusions}

In this paper, we generalize the induced $g$-noncommuting graph of a finite group $G$ by introducing the graph $\Delta_{H, G}^{g}$, where $H$ is a subgroup of $G$. We generalize certain results, namely (Lemma 2.4, [20]), (Lemma 3.1, [20]) and (Theorem 2.1, [21]) in Theorems 1, 6 and 7. In (Theorem 2.5, [22]), it was shown that $\Delta_{G, G}^{g}$ is not a tree. In Section 2, we consider the question whether $\Delta_{H, G}^{g}$ is a tree or not and we show that $\Delta_{H, G}^{g}$ is not a tree in general. In [21], Nasiri et al. showed that $\operatorname{diam}\left(\Delta_{G, G}^{g}\right) \leq 4$ if $\Delta_{G, G}^{g}$ is connected. Furthermore, they conjectured that $\operatorname{diam}\left(\Delta_{G, G}^{g}\right) \leq 2$ if $\Delta_{G, G}^{g}$ is connected. In Section 3, we show that this is not true in case of the graph $\Delta_{H, G}^{g}$, where $H$ is a proper subgroup of $G$. In particular, we 
identify a subgroup $H$ of $D_{2 n}$ in Theorem 12 such that $\operatorname{diam}\left(\Delta_{H, D_{2 n}}^{g}\right)=3$ while discussing connectivity and diameter of $\Delta_{H, D_{2 n}}^{g}$. It will be interesting to consider other families of finite groups (e.g., semidihedral groups and generalized quaternion groups) and find $\operatorname{diam}\left(\Delta_{H, G}^{g}\right)$.

Author Contributions: Investigation, M.S., R.K.N., Y.S.; writing—original draft preparation, M.S., R.K.N., Y.S.; writing - review and editing, M.S., R.K.N., Y.S. All authors have read and agreed to the published version of the manuscript.

Funding: This research received no external funding.

Institutional Review Board Statement: Not applicable.

Informed Consent Statement: Not applicable.

Data Availability Statement: Not applicable.

Acknowledgments: The first author would like to thank DST for the INSPIRE Fellowship. The authors are grateful to the referees for their valuable comments and suggestions.

Conflicts of Interest: The authors declare no conflict of interest.

\section{References}

1. Abdollahi, A.; Akbari, S.; Maimani, H.R. Non-commuting graph of a group. J. Algebra 2006, 298, 468-492. [CrossRef]

2. Afkhami, M.; Farrokhi, D.G.M.; Khashyarmanesh, K. Planar, toroidal and projective commuting and non-commuting graphs. Comm. Algebra 2015, 43, 2964-2970. [CrossRef]

3. Ahanjideh, N.; Iranmanesh, A. On the relation between the non-commuting graph and the prime graph. Int. J. Group Theory 2012, 1, 25-28.

4. Darafsheh, M.R. Groups with the same non-commuting graph. Discret. Appl. Math. 2009, 157, 833-837. [CrossRef]

5. Darafsheh, M.R.; Bigdely, H.; Bahrami, A.; Monfared, M.D. Some results on non-commuting graph of a finite group. Ital. J. Pure Appl. Math. 2010, 27, 107-118.

6. Dutta, P.; Nath, R.K. On Laplacian energy of non-commuting graphs of finite groups. J. Linear Top. Algebra 2018, 7, 121-132.

7. Dutta, P.; Dutta, J.; Nath, R.K. On Laplacian spectrum of non-commuting graphs of finite groups. Indian J. Pure Appl. Math. 2018, 49, 205-216. [CrossRef]

8. Jahandideh, M.; Darafsheh, M.R.; Sarmin, N.H.; Omer, S.M.S. Conditions on the edges and vertices of non-commuting graph. J. Tech. 2015, 74, 73-76. [CrossRef]

9. Jahandideh, M.; Darafsheh, M.R.; Shirali, N. Computation of topological indices of non-commuting graphs. Ital. J. Pure Appl. Math. 2015, 34, 299-310.

10. Jahandideh, M.; Modabernia, R.; Shokrolahi, S. Non-commuting graphs of certain almost simple groups. Asian-Eur. J. Math. 2019, 12, 1950081. [CrossRef]

11. Jahandideh, M.; Sarmin, N.H.; Omer, S.M.S. The topological indices of non-commuting graph of a finite group. Int. J. Pure Appl. Math. 2015, 105, 27-38. [CrossRef]

12. Moghaddamfar, A.R. About non-commuting graphs. Sib. Math. J. 2005, 47, 1112-1116.

13. Moghaddamfar, A.R.; Shi, W.J.; Zhou, W.; Zokayi, A.R. On the non-commuting graph associated with a finite group. Sib. Math. J. 2005, 46, 325-332. [CrossRef]

14. Nath, R.K.; Sharma, M.; Dutta, P.; Shang, Y. On $r$-noncommuting graph of finite rings. Axioms 2021, 10, 233. [CrossRef]

15. Talebi, A.A. On the non-commuting graphs of group $D_{2 n}$. Int. J. Algebra 2008, 2, 957-961.

16. Vatandoost, E.; Khalili, M. Domination number of the non-commuting graph of finite groups. Electron. J. Graph Theory Appl. 2018, 6, 228-237. [CrossRef]

17. Neumann, B.H. A problem of Paul Erdös on groups. J. Aust. Math. Soc. 1976, 21, 467-472. [CrossRef]

18. Sharma, M.; Nath, R.K. Relative g-Noncommuting Graph of Finite Groups. Available online: https://arxiv.org/pdf/2008.04123 .pdf (accessed on 10 September 2020).

19. Nasiri, M.; Erfanian, A.; Ganjali, M.; Jafarzadeh, A. g-noncommuting graph of some finite groups. J. Prime Res. Math. 2016, 12, 16-23.

20. Nasiri, M.; Erfanian, A.; Ganjali, M.; Jafarzadeh, A. Isomorphic g-noncommuting graphs of finite groups. Pub. Math. Deb. 2017, 91, 33-42. [CrossRef]

21. Nasiri, M.; Erfanian, A.; Mohammadian, A. Connectivity and planarity of $g$-noncommuting graphs of finite groups. J. Agebra Appl. 2018, 16, 1850107. [CrossRef]

22. Tolue, B.; Erfanian, A.; Jafarzadeh, A. A kind of non-commuting graph of finite groups. J. Sci. Islam. Repub. Iran 2014, 25, 379-384. 